Tropical Journal of Pharmaceutical Research November 2017; 16 (11): 2773-2776

ISSN: $1596-5996$ (print); 1596-9827 (electronic)

(c) Pharmacotherapy Group, Faculty of Pharmacy, University of Benin, Benin City, 300001 Nigeria.

All rights reserved.

Available online at http://www.tjpr.org

Original Research Article

http://dx.doi.org/10.4314/tjpr.v16i11.27

\title{
Potential protective role of hydrogen against cisplatin- induced side effects during chemotherapy: A mini-review of a novel hypothesis for antagonism of hydrogen
}

\author{
Fang $\mathrm{Li}^{1}$, Tao Li ${ }^{1 *}$, Chu-Rong $\mathrm{Li}^{1}$, Xiao-Li Yuan ${ }^{1}$, Bo Chen ${ }^{2}$, Jun Zhang ${ }^{1}$ and Jin- \\ Yi Lang ${ }^{1}$ \\ ${ }^{1}$ Department of Radiation Oncology, Sichuan Cancer Hospital, ${ }^{2}$ Department of Oncology, Chengdu First People's Hospital, \\ Chengdu 610041, China \\ ${ }^{*}$ For correspondence: Email: litaocdu@yeah.net; Tel: +86-28-85420173; Fax: +86-28-85420837
}

Sent for review: 3 October 2016

Revised accepted: 12 October 2017

\begin{abstract}
Purpose: To review the potential protective role of hydrogen against cisplatin-induced side effects during chemotherapy.

Methods: We searched PubMed and SCOPUS using the following keywords and combinations in titles, keywords, abstracts and full texts: cisplatin; side effects; chemotherapy; tumor; toxicity; hydrogen; reactive oxidative species; and ischemic reperfusion.

Results: The pathogenesis of cisplatin-induced side effects is suggested based on the increased level of reactive oxidative species (ROS). Cisplatin induces ROS-dependent platelet apoptosis via the extracellular signal-regulated kinase (ERK) signaling pathway, which might have contributed to cisplatininduced hematotoxicity, and in particular, thrombocytopenia. Molecular hydrogen has been shown to have therapeutic effects against damage to various organs (especially kidney, brain and liver) caused by ischemic reperfusion (IR) through selective elimination of the most cytotoxic ROS hydrogen radicals without affecting other types of ROS involved in signal transduction in vitro and in vivo.

Conclusion: Hydrogen may not only alleviate hematotoxicity in patients with hemorrhagic tendencies during cisplatin-based chemotherapy, but also has a potential protective effect against other side effects induced by cisplatin.
\end{abstract}

Keywords: Reactive oxygen species, Hydrogen radicals, Cisplatin, Hepatotoxicity, Chemotherapy, Side effects, Antagonism

Tropical Journal of Pharmaceutical Research is indexed by Science Citation Index (SciSearch), Scopus, International Pharmaceutical Abstract, Chemical Abstracts, Embase, Index Copernicus, EBSCO, African Index Medicus, JournalSeek, Journal Citation Reports/Science Edition, Directory of Open Access Journals (DOAJ), African Journal Online, Bioline International, Open-J-Gate and Pharmacy Abstracts

\section{INTRODUCTION}

Cis-diamminedichloroplatinum II (cisplatin) is one of the most effective platinum-based agents, and has broad spectrum anti-neoplastic effects on various tumors. Unfortunately, cisplatin-based chemotherapy is usually accompanied by several side effects, including nephrotoxicity, peripheral neuropathy, and hematologic toxicity [1], in which reactive oxidative species (ROS) play key roles [2].
Cisplatin induces the generation of various ROS, including hydroxyl radicals, hydrogen peroxide, and superoxide anions, in cells [3,4]. Hydroxyl radicals are the most cytotoxic oxidants that indiscriminately interact with lipids, proteins, and nucleic acids, thus leading to lipid peroxidation, inactivation of proteins, and DNA fragments $[3,4]$. Cisplatin induces ROS-mediated platelet apoptosis via the extracellular signal-regulated kinase (ERK) signaling pathway, which might 
contribute to cisplatin-induced hematotoxicity, and in particular, thrombocytopenia. Moreover, two anti-oxidants ( $\mathrm{N}$-acetylcysteine [NAC] and dithiothreitol [DTT]) effectively abolish ROS production, ERK activation, and platelet apoptosis elicited by cisplatin [2]. Although many anti-oxidants, such as NAC, vitamin E, vitamin C, allopurinol, erdosteine, edaravone, and melatonin, exhibit protective effects in animal models involving cisplatin treatment, high-dose anti-oxidants often cause other side effects and interfere with the anti-tumor effects of cisplatin in clinical applications [5-7]. Specifically, hydrogen peroxide and superoxide anion play key roles in cell proliferation, differentiation, and apoptosis as signaling molecules [3]. Therefore, more effective and safe agents are needed.

Molecular hydrogen has been shown to have therapeutic effects against damage to various organs, especially the kidneys, brain, and liver induced caused by ischemic reperfusion (I/R) through selective elimination of the most cytotoxic ROS hydrogen radicals without affecting other types of ROS involved in signal transduction in vitro and in vivo [8-23]. Specifically, Ohsawa and colleagues $[10,13,16,22]$ reported that molecular hydrogen selectively eliminates ROS levels, especially hydroxyl radicals, and exhibits protective effects in PC12 cultured cells, cell-free systems, and acute rat models of cerebral artery occlusion induced by focal ischemia and reperfusion. Furthermore, it has been reported that hydrogen inhibits ROS and oxidative stress-induced damage involving the hippocampus and learning tasks in chronic physically-restrained mouse models [16]. In addition, inhalation of hydrogen gas also inhibits liver injury caused by $\mathrm{I} / \mathrm{R}$ through selectively abolishing hydrogical radicals in a mouse model [15]. Moreover, the protective effects of hydrogen-rich water against nephrotoxicity induced by cisplatin has been demonstrated in rat models based on dynamic contrast-enhanced CT (DCF-CT) analysis [10].
These data indicate that the beneficial effects of molecular hydrogen can be used to attenuate the injuries of various organs caused by elevated ROS and oxidative stress.

This work is based on the hypothesize that hydrogen may not only alleviate hematotoxicity in patients with hemorrhagic tendencies during cisplatin-based chemotherapy, but also has potentially protective effects against other adverse side effects induced by cisplatin (Figure $1)$.

\section{METHODS}

The online database, PubMed and SCOPUS, were searched using the following keywords and combinations in titles, keywords, abstracts and full texts: cisplatin; side effects; chemotherapy; tumor; toxicity; hydrogen; reactive oxidative species; and ischemic reperfusion.

\section{RESULTS AND DISCUSSION}

Compared with other known treatments, transfusion of hydrogen may have more advantages in preventing cisplatin-induced adverse side effects in patients receiving cisplatin-based chemotherapy. We reviewed related articles, and summarized three main pieces of evidence in support of our hypothesis.

1) Hydrogen selectively eliminates the most cytotoxic ROS hydrogen radicals to generate innocuous water, but does not affect the other types of physiologically beneficial ROS involved in signal transduction and biological activities without compromising the anti-tumor effects of cisplatin [3]. Thus, hydrogen may not only alleviate hematotoxicity in patients with hemorrhagic tendencies during cisplatin-based chemotherapy, but also has potentially protective effects against other side effects incurred by cisplatin.

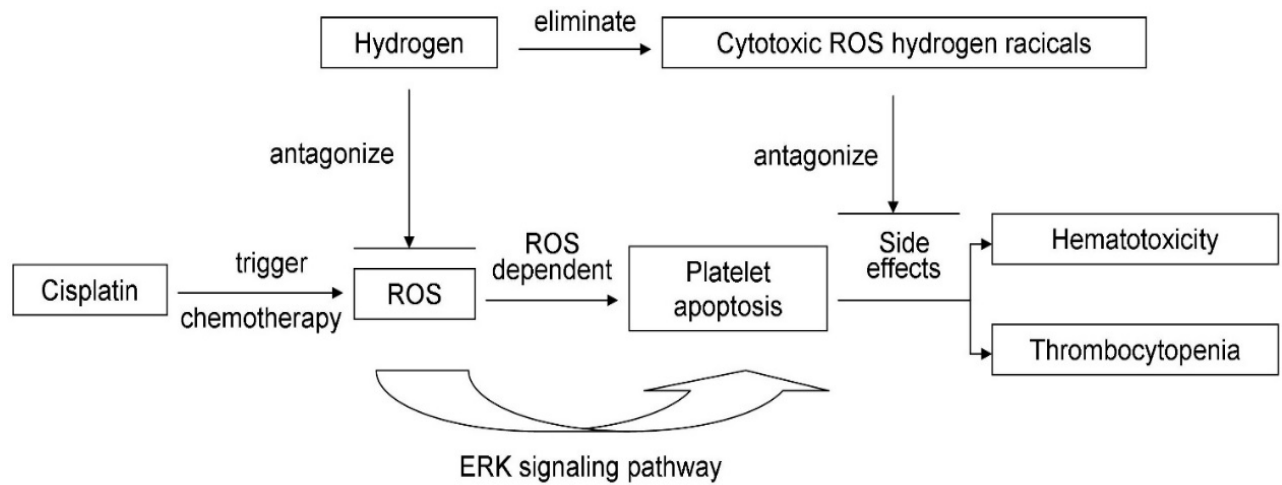

Figure 1: Hypothesis illustrating hydrogen antagonizing cisplatin-induced side effects in chemotherapy. ROS: reactive oxidative species; ERK: extracellular signal-regulated kinase 
2) As a mild but effective anti-oxidant, hydrogen is much smaller in weight than most known antioxidants, and is electronically neutral. Moreover, hydrogen can diffuse rapidly into tissues and cells, penetrate biomembranes, and diffuse into nuclei, the cytosol, and mitochondria, which are the main sources of ROS production, and selectively abolish hydroxyl radicals in living cells [8-23].

3) A hydrogen concentration of 1 or $2 \%$ shows an efficient protective effect on organ injuries in animal models, and hydrogen does not explore or burn at a concentration $<4.7 \%$.

4) Production of ROS is suggested in platelets during storage [24]. In fact, increasing periods without agitation induce platelet apoptotic cascades, including depolarization of MMP, down-regulation of $\mathrm{Bcl}-2$, up-regulation of $\mathrm{Bax}$ and Bak, activation of caspases, and PS exposure, which are closely correlated with elevation of intracellular ROS levels during storage $[25,26]$. Furthermore, thrombin elicits platelet apoptosis through the production of ROS $[27,28]$. Hydrogen may protect platelet apoptosis and improve the quality of platelets in hydrogen during storage. Therefore, hydrogen may have the potential to be used as a new class of antiapoptotic agents for platelet storage.

\section{CONCLUSION}

In conclusion, our review of the literature and existing data indicate that hydrogen not only inhibits cisplatin-induced hematotoxicity (especially bleeding), but also improves longlasting improvement in function of kidneys and other organs by attenuating oxidative stress, preventing platelet apoptosis, and providing protection against injured organs.

\section{DECLARATIONS}

\section{Acknowledgement}

None provided.

\section{Conflict of Interest}

No conflict of interest associated with this work.

\section{Contribution of Authors}

The authors declare that this work was done by the authors named in this article and all liabilities pertaining to claims relating to the content of this article will be borne by them.

\section{Open Access}

This is an Open Access article that uses a funding model which does not charge readers or their institutions for access and distributed under the terms of the Creative Commons Attribution License (http://creativecommons.org/licenses/by/ 4.0) and the Budapest Open Access Initiative (http://www.budapestopenaccessinitiative.org/rea d), which permit unrestricted use, distribution, and reproduction in any medium, provided the original work is properly credited.

\section{REFERENCES}

1. Brito $A B$, Reis $F$, de Souza $C A$, Vassallo J, Lima CS. Intracranial primary dural diffuse large $B$-cell lymphoma successfully treated with chemotherapy. Int J Clin Exp Med, 2014; 7: 456-460.

2. Zhang W, Zhao L, Liu J, Du J, Wang Z, Ruan C, Dai K. Cisplatin induces platelet apoptosis through the ERK signaling pathway. Thromb Res, 2012; 130: 81-91.

3. Baek SM, Kwon CH, Kim JH, Woo JS, Jung JS, Kim YK. Differential roles of hydrogen peroxide and hydroxyl radical in cisplatin-induced cell death in renal proximal tubular epithelial cells. J Lab Clin Med, 2013; 142: 178186.

4. Olas $B$, WachoWicz $B$. Role of reactive nitrogen species in blood platelet functions. Platelets, 2007; 18: 555-565.

5. Gokcimen A, Cim A, Tola HT, Bayram D, Kocak A, Ozgüner $F$, Ayata $A$. Protective effect of $N$ acetylcysteine, caffeic acid and vitamin $E$ on doxorubicin hepatotoxicity. Hum Exp Toxicol, 2007; 26: 519-525.

6. Chirino YI, Pedraza-Chaverri J. Role of oxidative and nitrosative stress in cisplatin-induced nephrotoxicity. Exp Toxicol Pathol, 2009; 61: 223-242.

7. Assis J, Pereira D, Gomes $M$, Marques D, Marques I, Nogueira $A$, Catarino $R$, Medeiros $R$. Influence of CYP3A4 genotypes in the outcome of serous ovarian cancer patients treated with first line chemotherapy: implication of a CYP3A4 activity profile. Int J Clin Exp Med, 2013; 6: 552-561.

8. Kitamura A, Kobayashi S, Matsushita T, Fujinawa $H$, Murase K. Experimental verification of protective effect of hydrogen-rich water against cisplatin-induced nephrotoxicity in rats using dynamic contrast-enhanced CT. Br J Radiol, 2010; 83: 509-514.

9. Matsushita $T$, Kusakabe $Y$. Investigation of protective effect of hydrogen-rich water against cisplatin-induced nephrotoxicity in rats using blood oxygenation leveldependent magnetic resonance imaging. Jpn J Radiol, 2011; 29: 503-512.

10. Nakashima-Kamimura N, Mori T, Ohsawa I, Asoh S, Ohta S. Molecular hydrogen alleviates nephrotoxicity induced by an anti-cancer drug cisplatin without compromising anti-tumor activity in mice. Cancer Chemother Pharmacol, 2009; 64: 753-761. 
11. Nishioka A, Ogawa $Y$, Miyatake $K$, Tadokoro M, Nogami M, Hamada N, Kubota K, Kariya S, Kohsaki T, Saibara $T$, Okabayashi T, Hanazaki K. Safety and efficacy of image-guided enzyme-targeting radiosensitization and intraoperative radiotherapy for locally advanced unresectable pancreatic cancer. Oncol Lett, 2014; 8: 404-408.

12. Kawamura T, Huang CS, Tochigi N, Lee S, Shigemura N, Billiar TR, Okumura M, Nakao A, Toyoda Y. Inhaled hydrogen gas therapy for prevention of lung transplantinduced ischemia/reperfusion injury in rats. Transplantation, 2010; 90: 1344-1351.

13. Ohsawa I, Ishikawa $M$, Takahashi K, Watanabe $M$, Nishimaki K, Yamagata K, Katsura K, Katayama Y, Asoh S, Ohta S. Hydrogen acts as a therapeutic antioxidant by selectively reducing cytotoxic oxygen radicals. Nature Med, 2007; 13: 688-694.

14. Wood KC, Gladwin MT. The hydrogen highway to reperfusion therapy. Nature Med, 2007; 13: 673-674.

15. Fukuda K, Asoh S, Ishikawa M, Yamamoto Y, Ohsawa I, Ohta S. Inhalation of hydrogen gas suppresses hepatic injury caused by ischemia/reperfusion through reducing oxidative stress. Biochem Biophys Res Commun, 2007; 361: 670-674.

16. Nagata K, Nakashima-Kamimura N, Mikami T, Ohsawa I, Ohta S. Consumption of molecular hydrogen prevents the stress-induced impairments in hippocampusdependent learning tasks during chronic physical restraint in mice. Neuropsychopharmacology, 2009; 34 501-508.

17. Sato $Y$, Kajiyama S, Amano A, Kondo $Y$, Sasaki $T$, Handa S, Takahashi R, Fukui M, Hasegawa G, Nakamura N, Fujinawa $H$, Mori T, Ohta M, Obayashi $H$, Maruyama N, Ishigami A. Hydrogen-rich pure water prevents superoxide formation in brain slices of vitamin C-depleted SMP30/GNL knockout mice. Biochem Biophys Res Commun, 2008; 375: 346-350.

18. Gezer U, Keskin S, Igci A, Tukenmez M, Tiryakioglu D, Cetinkaya M, Disci R, Dalay N, Eralp Y. Abundant circulating microRNA in breast cancer patients fluctuate considerably during neoadjuvant chemotherapy. Oncol Lett, 2014; 8: 845-848.

19. Cai J, Kang Z, Liu K, Liu WW, Li RP, Zhang JH, Luo X, Sun $X$. Neuroprotective effects of hydrogen saline in neonatal hypoxia-ischemia rat model. Brain Res 2009; 1256: 129-137.

20. Sun $Q$, Cai J, Liu S, Liu $Y, X u W$, Tao $H$, Sun $X$. Hydrogen-rich saline provides protection against hyperoxic lung injury. J Surg Res, 2011; 165: e43-e49.

21. Ge $M$, Chi $X$, Zhang A, Luo G, Sun G, Xie H, Hei $Z$. Intestinal NF-E2-related factor-2 expression and antioxidant activity changes in rats undergoing orthotopic liver autotransplantation. Oncol Lett, 2013; 6: 1307-1312.

22. Hayashida K, Sano M, Ohsawa I, Shinmura K, Tamaki K, Kimura K, Endo J, Katayama T, Kawamura A, Kohsaka S, Makino S, Ohta S, Ogawa S, Fukuda K. Inhalation of hydrogen gas reduces infarct size in the rat model of myocardial ischemia-reperfusion injury. Biochem Biophys Res Commun, 2008; 373: 30-35.

23. Cai J, Kang Z, Liu WW, Luo X, Qiang S, Zhang JH, Ohta $S$, Sun $X, X u W$, Tao H, Li R. Hydrogen therapy reduces apoptosis in neonatal hypoxia-ischemia rat model. Neurosci Lett, 2008; 441: 167-172.

24. Sener A, Ozsavci D, Oba R, Demirel GY, Uras $F$, Yardimci KT. Do platelet apoptosis, activation, aggregation, lipid peroxidation and platelet-leukocyte aggregate formation occur simultaneously in hyperlipidemia? Clin Biochem, 2005; 38: 1081-1087.

25. Sandgren $P$, Stjepanovic A. High-yield platelet units revealed immediate $\mathrm{pH}$ decline and delayed mitochondrial dysfunction during storage in 100\% plasma as compared with storage in SSP+. Vox Sang, 2012; 103: 55-63.

26. Lu X, Li C, Wang YK, Jiang K, Gai XD. Sorbitol induces apoptosis of human colorectal cancer cells via p38 MAPK signal transduction. Oncol Lett, 2014; 7: 19921996.

27. Lopez JJ, Salido GM, Gomez-Arteta E, Rosado JA, Pariente JA. Thrombin induces apoptotic events through the generation of reactive oxygen species in human platelets. J Thromb Haemost 2007; 5:1283-1291.

28. Jiang L, Wang L, Chen L, Cai GH, Ren QY, Chen JZ, Shi $\mathrm{HJ}$, Xie YH. As2O3 induces apoptosis in human hepatocellualr carcinoma HepG2 cells through a ROSmediated mitochondrial pathway and activation of caspases. Int J Clin Exp Med 2015; 8: 2190-2196. 